\title{
Coleoptera (Arthropoda, Insecta) Associados às Copas de Attalea phalerata Mart. (Arecaceae) no Pantanal de Poconé, Mato Grosso, Brasil
}

\author{
Leandro Dênis Battirola ${ }^{\bowtie}$, Geane Brizzola dos Santos ${ }^{1}$, Germano Henrique Rosado-Neto² \& $^{2}$ \\ Marinêz Isaac Marques ${ }^{1}$
}

1. Universidade Federal de Mato Grosso, e-mail: ldbattirola@uol.com.br (Autor para correspondência ${ }^{\bowtie}$ ), gbrizola@yahoo.com.br, marinez513@gmail.com. 2. Universidade Federal do Paraná, e-mail: rosadoneto@ufpr.br.

\section{EntomoBrasilis 7 (1): 20-28 (2014)}

Resumo. As palmeiras correspondem a um importante elemento nos ecossistemas tropicais, servindo como fonte alimentar e habitat para uma grande variedade de organismos invertebrados e vertebrados. Considerando o papel destas plantas como hospedeiras de diferentes espécies este estudo objetivou avaliar de maneira descritiva a composição, distribuição em guildas tróficas e a biomassa da comunidade de Coleoptera associada às copas de Attalea phalerata Mart. (Arecaceae) no Pantanal de Poconé, Mato Grosso. Ao todo foram amostrados seis indivíduos desta palmeira em fevereiro 2001, empregando-se a metodologia de termonebulização de copas. Obtiveram-se 7.670 indivíduos $\left(77,5\right.$ ind./ $\left.\mathrm{m}^{2}\right)$, sendo 5.044 adultos (65,7\%; 50,9 ind. $\left./ \mathrm{m}^{2}\right)$ e 2.626 larvas $\left(34,3 \% ; 26,5\right.$ ind. $\left./ \mathrm{m}^{2}\right)$. Os adultos foram distribuídos em 43 famílias e 467 morfoespécies. As famílias mais representativas foram Endomychidae, Nitidulidae, Tenebrionidae, Staphylinidae e Curculionidae, correspondendo a $66,9 \%$ do total coletado. Dentre as guildas tróficas, saprófagos, fungívoros e herbívoros predominaram sobre predadores. A maior riqueza de espécies foi observada em Staphylinidae, Curculionidae, Tenebrionidae e Chrysomelidae. A maior biomassa foi registrada para Scarabaeidae e Tenebrionidae, seguidos por Nitidulidae e Curculionidae. Esses resultados indicam que a copa de A. phalerata é habitat para uma grande diversidade de Coleoptera, bem como local de reprodução, fato evidenciado pelo elevado número de larvas amostradas neste estudo.

Palavras-chave: Áreas inundáveis; besouros; palmeiras; pulso de inundação.

\section{Coleoptera (Arthropoda, Insecta) Associated with the Canopies of Attalea phalerata Mart. (Arecaceae) in the Pantanal of Poconé, Mato Grosso, Brazil}

Abstract. The palm trees correspond to an important element in tropical ecosystems, serving as food source and habitat for a wide variety of invertebrates and vertebrates. Considering the role of these plants as hosts of different species, this study evaluated descriptively the composition, trophic guilds and biomass of the community of Coleoptera associated with canopies Attalea phalerata Mart. (Arecaceae) in the Pantanal of Poconé, Mato Grosso. Six palm trees were sampled during the high water season of the Pantanal of Mato Grosso (February 2001), by canopy fogging. A total of 7,670 specimens were collected $\left(77.5 \mathrm{ind} . / \mathrm{m}^{2}\right), 5,044$ adults $\left(65.7 \% ; 50.9 \mathrm{ind} . / \mathrm{m}^{2}\right)$ and 2,626 larvae $\left(34.3 \% ; 26.5 \mathrm{ind} . / \mathrm{m}^{2}\right)$. Adults $(65.7 \%)$ represented 43 families and 467 morphospecies. The dominant families were Endomychidae, Nitidulidae, Tenebrionidae, Staphylinidae and Curculionidae, representing $66.9 \%$ of the total catch. Saprophages, fungivores and herbivores prevailed over predators. Highest richness of species was found for Staphylinidae, Curculionidae, Tenebrionidae and Chrysomelidae. Highest biomass was found in Scarabaeidae and Tenebrionidae followed by Nitidulidae and Curculionidae. These results indicate that the canopy of $A$. phalerata is habitat for a wide variety of Coleoptera, as well a reproduction site, as evidenced by the high number of larvae sampled in this study.

Keywords: Beetles; flood pulse; palms; wetlands.

(1) $\mathrm{s}$ ecossistemas tropicais, devido à elevada diversidade biológica, caracterizam-se pela complexidade de suas relações ecológicas. Nestas áreas, as palmeiras representam importantes elementos da paisagem, que além de servirem como hospedeiras para inúmeros grupos de artrópodes constituem confiável fonte de recursos alimentares aos mesmos, em diferentes estágios de sua fenologia (Howard 2001; SANTos et al. 2003; BatTirola et al. 2004a, 2005, 2007; Gurgel-GonÇALVES et al. 2006).

Dentre os táxons que possuem íntima associação com estas plantas, destacam-se os Coleoptera, pois atuam como polinizadores de diversas espécies, predadores de espécies praga, consumidores de frutos ou as utilizam como hospedeira (GIBLINDAVIS 2001; MOORE 2001; Cysne et al. 2013). Tais associações são possíveis já que os coleópteros atuam em diferentes níveis tróficos em virtude de seus múltiplos hábitos alimentares, participando de diferentes processos ecológicos nos ecossistemas naturais (ERWin \& SCOTT 1980; Stork 1987; EndRess 1994).
Dentre os estudos ecológicos desenvolvidos com Coleoptera, a investigação sobre as formas de utilização, distribuição e preferência de habitat em diferentes estratos florestais tem sido conduzida (DAvies et al. 1997; WAGNER 1997; Charles \& BASSET 2005; Gonçalves \& Louzada 2005). Diversos grupos dentre os Coleoptera podem utilizar diferentes habitats ao longo do ciclo de vida, demonstrando, portanto, hábitos alimentares variados entre as fases larval e adulta como Chrysomelidae e Curculionidae que possuem espécies com desenvolvimento larval no solo, alimentando-se, principalmente, de raízes ou matéria em decomposição, e durante a fase adulta vivem, geralmente, associados à espécies vegetais (ADIs 1988), com ou não especificidade hospedeira (BASSET et al. 1996; BASSET \& SAMUELSON 1996; Novotny et al. 1999, 2002a, 2002b; Роком et al. 2005).

Agências de Financiamento: Instituto Max-Planck para Limnologia, Bundesministerium für Bildung, Wissenschaft Forschung und Technologie (BMBF) e CNPq. 
T Inúmeros são os estudos em copas de árvores que objetivaram conhecer a composição das comunidades de Coleoptera (ADIS et al. 1984; HaMmond et al. 1997; Floren \& LinsenMair 1998a; MARQUES et al. 2001, 2006, 2007; WAGNER 2000, 2003; SANTOS et al. 2003; ØDEGAARD 2003). Além da composição das comunidades, diversos aspectos ecológicos referentes aos Coleoptera foram analisados como diversidade e similaridade da fauna entre locais e hospedeiros (DAvies et al. 1997), especificidade hospedeira e especialização efetiva (ERwin \& SCOTT 1980; MAwdSLEY \& STORK 1997), determinantes da riqueza de espécies (Allison et al. 1997; Kitching et al. 1997), diversidade e padrões de recolonização (FLOREN \& LinsENMAIR 1997, 1998b), comparação entre diferentes tipos de florestas e espécies de árvores (ERwin 1983; BARONE 2000; ADIs et al. 2011), relação entre tamanho do corpo e riqueza de espécies (BASSET et al. 1994; BASSET 1997) e tamanho do corpo e biomassa (STORK \& BLACKBURN 1993).

Especificamente, no Brasil, as pesquisas sobre Coleoptera em copas foram realizadas, principalmente, na Amazônia (ADIs 1982, 1997; ERwIN \& AdIs 1982; ERwIN 1983; AdIs et al. 2011) e em áreas de vegetação monodominante no Pantanal matogrossense (MARQUES et al. 2001, 2006, 2007; SANTOS et al. 2003). Considerando a importância destes insetos na estruturação das comunidades de artrópodes associadas ao dossel de florestas, este estudo objetivou analisar de maneira descritiva a composição, distribuição em guildas tróficas e biomassa da comunidade de Coleoptera adultos associada à copa de Attalea phalerata Mart. (Arecaceae) em áreas que esta palmeira forma adensamentos monodominantes, no Pantanal de Poconé, Mato Grosso, contribuindo para o conhecimento da diversidade regional.

\section{MATERIAL E MÉTODOS}

Área de estudo. Esse estudo foi realizado no Pantanal de Cuiabá-Bento Gomes-Paraguaizinho, denominado Pantanal de Poconé, na localidade de Pirizal ( $16^{\circ} 15^{\prime}$ e $17^{\circ} 54^{\prime}$ S, $56^{\circ} 36^{\prime}$ e $57^{\circ} 56^{\prime}$ W) município de Nossa Senhora do Livramento, Mato Grosso. Esta região caracteriza-se por apresentar estações bem definidas, com o período chuvoso entre outubro e abril, e as inundações periódicas entre dezembro e março (o,6-1,5 m de altura), com quatro períodos sazonais distintos (cheia, vazante, seca e enchente) (HEckMan 1998). Este estudo foi conduzido em áreas em que $A$. phalerata forma adensamentos monodominantes denominados, regionalmente, como acurizais, geralmente, associados a áreas arenosas que não sofrem inundações, apesar de se localizarem as margens destas áreas. Embora as coletas tenham ocorrido durante o período de cheia na região norte do Pantanal de Mato Grosso (fevereiro de 2001), o acurizal estudado não foi atingido pela inundação.

Metodologia. Seis indivíduos de A.phalerata foram nebulizados empregando-se o método de termonebulização de copas ("canopy fogging") em fevereiro de 2001, utilizando-se piretróide sintético, Lambdacialotrina a 0,5\%. Para a seleção das palmeiras seguiram-se os critérios propostos por Adis et al. (1998), e para os procedimentos de nebulização e coletas BATTiRola et al. (2004a). Para a coleta todas as palmeiras tiveram seu diâmetro na base circundado por 16-17 funis de nylon (1 m de diâmetro cada), de acordo com a abrangência da copa. Ao todo foram analisados 99 $\mathrm{m}^{2}$ de área de copas.

Os indivíduos adultos de Coleoptera foram identificados ao nível taxonômico de família, seguindo a classificação proposta por LAWRENCE et al. (2000) e, posteriormente, morfoespeciados e agrupados em guildas tróficas de acordo com ARNETT (1963), Erwin (1983) e Hammond et al. (1996), com ênfase em Erwin (1983) por ser específica para este tipo de habitat. As larvas foram apenas quantificadas em nível de Ordem. Os Curculionidae foram identificados conforme Alonso-ZARAZAGA \& LyAL (1999) e MARVALDI \& LANTERI (2005) e a morfoespeciação foi efetuada com base na Coleção de Referência do Laboratório de Ecologia e Taxonomia de Artrópodes (LETA) do Instituto de Biociências - Universidade
Federal de Mato Grosso, específica para o Pantanal, e confirmada por taxonomistas.

A riqueza de espécies foi avaliada empregando-se os estimadores Jackknife 1 e Bootstrap, calculados pelo programa EstimateS, versão 6.o b1 (Colwell 2000). As amostras das palmeiras I, IV e V foram sorteadas para a avaliação da biomassa da comunidade e a sua distribuição dentre os diferentes grupos tróficos de Coleoptera. Os indivíduos foram analisados por palmeira amostrada e secos a $60^{\circ} \mathrm{C}$ em estufa, até que o peso se estabilizasse, e em seguida pesados em balança analítica de precisão de o,01 mg (Kern 410), e preservados em álcool a 92\%. A distribuição da biomassa dentre os níveis tróficos foi avaliada pela análise de variância $(\alpha=0,5)$, através do Programa SPSS versão 12.0 (2006).

\section{RESULTADOS}

Um total de 7.670 indivíduos de Coleoptera (77,5 ind./ $\mathrm{m}^{2}$ ), 5.044 adultos (50,9 ind./ $\left.\mathrm{m}^{2} ; 65,7 \%\right)$ e 2.626 larvas $(26,5$ ind./m²; $34,3 \%$ ) foi obtido em copas de seis indivíduos de $A$. phalerata. Os 5.044 indivíduos adultos estão distribuídos em 43 famílias e 467 morfoespécies. Endomychidae (11,7 ind./ $\left.\mathrm{m}^{2} ; 22,9 \%\right)$, Nitidulidae $\left(8,1\right.$ ind. $\left./ \mathrm{m}^{2} ; 15,8 \%\right)$, Tenebrionidae $\left(6,0\right.$ ind. $\left./ \mathrm{m}^{2} ; 11,7 \%\right)$, Staphylinidae (3,6 ind./ $\left./ \mathrm{m}^{2} ; 9,4 \%\right)$ e Curculionidae $\left(3,6\right.$ ind. $/ \mathrm{m}^{2}$; $7,1 \%$ ) foram os táxons mais representativos na amostragem geral de Coleoptera, correspondendo a $66,9 \%$ do total de indivíduos coletados (Tabela 1).

Com relação à riqueza de espécies, Staphylinidae (102 spp.; 21,8\%), Curculionidae (81 spp.; 17,3\%), Tenebrionidae (33 spp.; 7,0\%) e Chrysomelidae (29 spp.; 6,2\%) representaram 52,3\% da riqueza total. Apesar de Endomychidae e Nitidulidae serem dominantes em número de indivíduos possuem apenas $10 \mathrm{e}$ 14 morfoespécies, respectivamente, demonstrando uma baixa riqueza neste habitat. Dentre as 43 famílias identificadas, apenas $16(37,2 \%)$ foram amostradas em todas as copas, 16 apareceram com frequências intermediárias, ocorrendo entre três e cinco palmeiras, e 11 ocorreram em menos de três palmeiras (Tabela 1).

Os Curculionidae corresponderam a 7,1\% do total de Coleoptera adultos capturados (358 indivíduos; 3,6 ind./ $\mathrm{m}^{2}$ ) (Tabela 2) e estão representados por 81 espécies em 13 subfamílias, sendo Scolytinae a mais representativa (178 ind.; 49,7\%). Andranthobius sp.2 (Curculioninae, Derelomini) foi a morfoespécie mais abundante (58 ind.; 16,2\%), seguida por Derelomini sp.10 (11 ind.; 3,1\%) e Derelomini sp.21 (8 ind.; 2,2\%). Scolytinae também apresentou a maior riqueza de espécies (22 spp.), seguida por Curculioninae (19 spp.) e Baridinae (9 spp.) (Tabela 2).

Com relação à riqueza de espécies, o estimador Bootstrap demonstrou que $82,3 \%$ das espécies foram amostradas, sendo 567 espécies esperadas ao contrário das 467 obtidas. Já o estimador Jackknife 1, apontou que 66,6\% das espécies foram obtidas, sendo 234 espécies esperadas a mais na amostragem (Figura 1). Dentre as 467 morfoespécies identificadas, 208 $(44,5 \%)$ são espécies raras "singletons", com um único indivíduo amostrado, e 68 (14,5\%) são "doubletons", com apenas dois indivíduos amostrados. A grande quantidade de indivíduos pouco abundantes é visível na curva de abundância das espécies (Figura 2). Staphylinidae (54 espécies), Curculionidae (45 espécies) e Chrysomelidae (22 espécies) contribuíram com o maior número de espécies "singletons".

Quatro guildas tróficas foram definidas para a comunidade de Coleoptera conforme a classificação de ERWIN (1983) e, com base na abundância de indivíduos, verifica-se a predominância de saprófagos (28,5\%), fungívoros (27,6\%) e herbívoros $(26,7 \%)$ sobre predadores $(17,2 \%)$ (Tabela 1 , Figura $3 \mathrm{~A})$. Os saprófagos dominantes foram Nitidulidae (797 ind.; 8,1 ind./m²) e Tenebrionidae (510 ind.; 6,o ind./ $\mathrm{m}^{2}$ ) que representaram $91,1 \%$ da abundância total desta guilda. Endomychidae (1.156 ind.; 11,7 ind. $/ \mathrm{m}^{2}$ ) foi predominante dentre os fungívoros, correspondendo

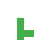


Tabela 1. Número de indivíduos coletados (adultos e larvas) (N), abundância relativa (\%), frequência de ocorrência por palmeira (\%), riqueza (S) e guildas tróficas das famílias de Coleoptera (adultos) obtidas em seis copas de A. phalerata Mart. (Arecaceae) no Pantanal de Poconé, Mato Grosso. *P = predadores, $\mathrm{H}$ = herbívoros, $\mathrm{S}$ = saprófagos, $\mathrm{F}$ = fungívoros, $\mathrm{D}=$ decompositores e $\mathrm{X}$ = xilófagos, () = Hábito de nutrição considerado secundário.

\begin{tabular}{|c|c|c|c|c|c|c|c|c|c|c|c|}
\hline \multirow[t]{2}{*}{ Família } & \multicolumn{6}{|c|}{ Palmeiras } & \multirow{2}{*}{$\begin{array}{c}\text { Total } \\
(\mathrm{N})\end{array}$} & \multirow{2}{*}{$\begin{array}{l}\text { Abundância } \\
\text { Relativa (\%) }\end{array}$} & \multirow{2}{*}{$\begin{array}{c}\text { Frequência } \\
\text { (\%) }\end{array}$} & \multirow{2}{*}{$\begin{array}{c}\text { Riqueza } \\
\text { (S) }\end{array}$} & \multirow{2}{*}{\begin{tabular}{c|} 
Guilda \\
Trófica*
\end{tabular}} \\
\hline & I & II & III & IV & $\mathbf{V}$ & VI & & & & & \\
\hline Anobiidae & 32 & 20 & 22 & 79 & 97 & 14 & 264 & 5,2 & 100,0 & 10 & $\mathrm{H}$ \\
\hline Anthicidae & 2 & - & - & 4 & 1 & 5 & 12 & 0,2 & 66,6 & 4 & $\mathrm{~S}$ \\
\hline Biphyllidae & 10 & - & - & 2 & - & 2 & 14 & 0,3 & 50,0 & 2 & $\mathrm{~F}$ \\
\hline Bostrichidae & - & - & - & 1 & - & - & 1 & $<0,1$ & 16,6 & 1 & $\mathrm{~S}$ \\
\hline Bothrideridae & 1 & - & - & 1 & - & - & 2 & $<0,1$ & 33,3 & 1 & $\mathrm{P}$ \\
\hline Brentidae (Apioninae) & 1 & 1 & - & - & 1 & - & 3 & 0,1 & 50,0 & 2 & $\mathrm{H}$ \\
\hline Buprestidae & 1 & - & - & - & - & 1 & 2 & $<0,1$ & 33,3 & 2 & $\mathrm{H}$ \\
\hline Carabidae & 22 & 2 & 4 & 22 & 19 & 29 & 98 & 1,9 & 100,0 & 15 & $\mathrm{P}$ \\
\hline Cerambycidae & - & 5 & 3 & 1 & 4 & 3 & 16 & 0,3 & 83,3 & 9 & $\mathrm{H}$ \\
\hline Chrysomelidae & 6 & 4 & 2 & 5 & 13 & 25 & 55 & 1,1 & 100,0 & 29 & $\mathrm{H}$ \\
\hline (Bruchinae) & (1) & - & - & - & (5) & (11) & (17) & $(0,3)$ & $(50,0)$ & (2) & $\mathrm{H}$ \\
\hline Ciidae & 1 & - & 1 & 6 & 1 & 6 & 15 & 0,3 & 83,3 & 5 & $\mathrm{~F}$ \\
\hline Cleridae & - & 1 & - & 3 & 2 & 2 & 8 & 0,2 & 66,6 & 6 & $\mathrm{P}$ \\
\hline Coccinellidae & 49 & 30 & 16 & 17 & 9 & 5 & 126 & 2,5 & 100,0 & 9 & $\mathrm{P}$ \\
\hline Colydiidae & 4 & 1 & - & 8 & 2 & 18 & 33 & 0,7 & 100,0 & 6 & $\mathrm{P}(\mathrm{F})$ \\
\hline Corylophidae & 16 & 7 & 7 & 16 & 8 & 30 & 84 & 1,7 & 100,0 & 15 & $\mathrm{P}$ \\
\hline Cucujidae & - & 5 & - & 5 & 1 & 2 & 13 & 0,3 & 66,6 & 4 & $\mathrm{P}(\mathrm{F})$ \\
\hline Curculionidae & 41 & 22 & 14 & 130 & 36 & 115 & 358 & 7,1 & 100,0 & 81 & $\mathrm{H}$ \\
\hline (Platypodinae) & - & - & - & - & (1) & (3) & (4) & $(0,1)$ & $(33,3)$ & (3) & $\mathrm{F}$ \\
\hline (Scolytinae) & (20) & (13) & (12) & (35) & (22) & (76) & (178) & $(3,5)$ & $(100,0)$ & (22) & $\mathrm{H}(\mathrm{F})$ \\
\hline Dytiscidae & - & 1 & - & - & - & - & 1 & $<0,1$ & 16,6 & 1 & $\mathrm{P}$ \\
\hline Elateridae & 5 & - & 1 & 1 & 5 & 15 & 27 & 0,5 & 83,3 & 11 & $\mathrm{H}(\mathrm{P})$ \\
\hline Endomychidae & 196 & 34 & 50 & 314 & 109 & 453 & 1156 & 22,9 & 100,0 & 10 & $\mathrm{~F}$ \\
\hline Histeridae & 8 & - & 1 & 4 & 1 & 3 & 17 & 0,3 & 83,3 & 7 & $\mathrm{P}$ \\
\hline Hydraenidae & 2 & 1 & - & - & - & 1 & 4 & 0,1 & 50,0 & 3 & $\mathrm{P}$ \\
\hline Lampyridae & - & - & - & 1 & 6 & - & 7 & 0,1 & 33,3 & 3 & $\mathrm{P}$ \\
\hline Lathridiidae & 45 & 6 & 3 & 14 & 34 & 50 & 152 & 3,0 & 100,0 & 5 & $\mathrm{~F}$ \\
\hline Leiodidae & - & - & - & - & - & 2 & 2 & $<0,1$ & 16,6 & 1 & $\mathrm{~S}$ \\
\hline Limnichidae & - & 1 & 3 & - & 1 & - & 5 & 0,1 & 50,0 & 2 & $?$ \\
\hline Limulodidae & 1 & - & - & - & 1 & - & 2 & $<0,1$ & 33,3 & 2 & $\mathrm{~S}$ \\
\hline Meloidae & 15 & 10 & 1 & 6 & 13 & 7 & 52 & 1,0 & 100,0 & 11 & $\mathrm{H}$ \\
\hline Monommidae & 1 & 4 & - & 3 & 4 & 10 & 22 & 0,4 & 83,3 & 4 & $\mathrm{H}$ \\
\hline Mordellidae & 2 & - & - & - & - & - & 2 & $<0,1$ & 83,3 & 1 & $\mathrm{H}$ \\
\hline Nitidulidae & 5 & 4 & 16 & 708 & 12 & 52 & 797 & 15,8 & 100,0 & 14 & $\mathrm{H}$ \\
\hline Phalacridae & 67 & 59 & 7 & 26 & 7 & 123 & 289 & 5,7 & 100,0 & 15 & $\mathrm{H}$ \\
\hline Ptiliidae & 22 & 10 & 11 & 39 & 10 & 18 & 110 & 2,2 & 100,0 & 18 & $\mathrm{~S}(\mathrm{~F})$ \\
\hline Ptilodactylidae & 2 & - & - & - & 2 & 2 & 6 & 0,1 & 50,0 & 2 & $\mathrm{H}$ \\
\hline Rhizophagidae & - & - & - & 5 & - & 2 & 7 & 0,1 & 33,3 & 1 & $\mathrm{P}$ \\
\hline Scaphidiidae & 1 & - & - & 4 & 2 & 1 & 8 & 0,2 & 66,6 & 2 & $\mathrm{~F}$ \\
\hline Scarabaeidae & 37 & 13 & 7 & 16 & 51 & 48 & 172 & 3,4 & 100,0 & 8 & $\mathrm{H}(\mathrm{S})$ \\
\hline Scydmaenidae & 5 & - & 1 & 2 & 16 & 6 & 30 & 0,6 & 83,3 & 7 & $\mathrm{P}$ \\
\hline Silvanidae & - & - & - & - & 1 & 1 & 2 & $<0,1$ & 33,3 & 1 & $\mathrm{~F}$ \\
\hline Staphylinidae & 99 & 31 & 46 & 97 & 96 & 107 & 476 & 9,5 & 100,0 & 102 & $\mathrm{P}(\mathrm{S}, \mathrm{F})$ \\
\hline (Pselaphinae) & (7) & (3) & (1) & (10) & (7) & (11) & (39) & $(0,8)$ & $(100,0)$ & 13 & $\mathrm{~F}$ \\
\hline Tenebrionidae & 87 & 76 & 23 & 78 & 161 & 167 & 592 & 11,7 & 100,0 & 33 & $\mathrm{~S}(\mathrm{~F})$ \\
\hline (Alleculinae) & $(11)$ & (5) & (1) & (17) & (21) & (27) & $(82)$ & $(1,6)$ & $(100,0)$ & (4) & $\mathrm{H}$ \\
\hline Throscidae & - & - & - & - & 1 & - & 1 & $<0,1$ & 16,6 & 1 & $\mathrm{H}$ \\
\hline Trogossitidae & 1 & - & - & - & - & - & 1 & $<0,1$ & 16,6 & 1 & $\mathrm{P}(\mathrm{S})$ \\
\hline Total adultos & 787 & 348 & 239 & 1.618 & 727 & 1.325 & 5.044 & 100 & - & 467 & - \\
\hline Total larvas & 401 & 188 & 56 & 1.510 & 113 & 358 & 2.626 & - & - & - & - \\
\hline Total geral & 1.188 & 536 & 295 & 3.128 & 840 & 1.683 & 7.670 & 100 & - & 467 & - \\
\hline
\end{tabular}




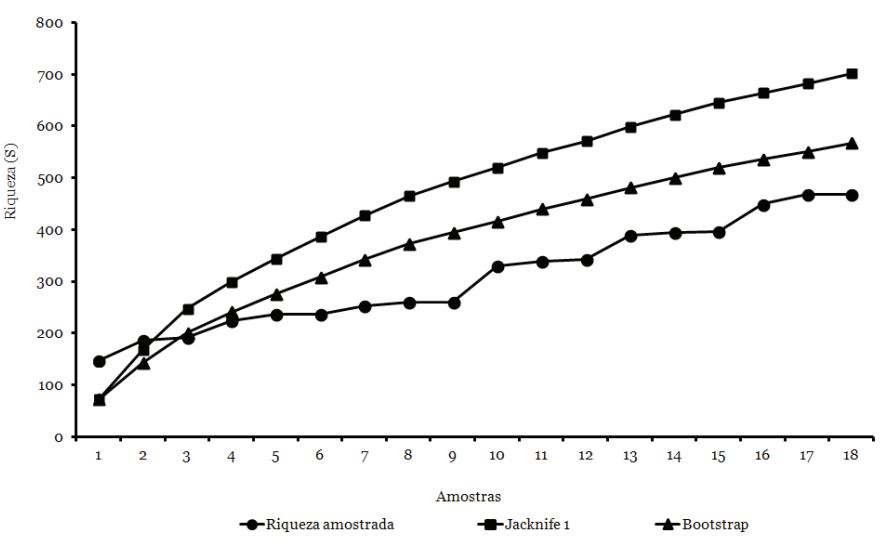

Figura 1. Comparação entre a riqueza de espécies obtida e a riqueza estimada, Bootstrap e Jacknife 1, para a comunidade de Coleoptera (adultos) associada a copa de A. phalerata Mart. (Arecaceae), durante a fase aquática do Pantanal de Mato Grosso.

a $83,2 \%$ do total deste agrupamento. Os herbívoros não apresentaram um único táxon dominante, sendo Curculionidae, excluindo-se Platypodinae (354 ind.; 3,6 ind./ $\mathrm{m}^{2}$ ), Phalacridae (289 ind.; 2,9 ind. $/ \mathrm{m}^{2}$ ) e Anobiidae (264 ind.; 2,7 ind. $/ \mathrm{m}^{2}$ ) os que mais se destacaram, compreendendo $67,4 \%$ do total desta guilda (Tabela 1).

Com relação à riqueza de espécies observou-se que herbívoros (183 spp.; 39,3\%) e predadores (168 spp.; 36,0\%) predominaram sobre saprófagos (73 spp.; 15,7\%) e fungívoros (41 spp.; 9,0\%) (Tabela 1, Figura 3B). Verifica-se que os grupos tróficos mais abundantes (saprófagos e fungívoros) possuem baixa riqueza quando comparados aos grupos tróficos menos abundantes, herbívoros e predadores que são mais ricos em espécies, demonstrando que a comunidade é estruturada por um número reduzido de espécies com elevada densidade populacional.

Os herbívoros que contribuíram com o maior número de espécies foram Curculionidae (78 spp.; 42,6\%), Chrysomelidae (29 spp.; $15,8 \%$ ) e Phalacridae (15 spp.; 8,2\%), que juntos corresponderam a $66,6 \%$ do total de morfoespécies para essa guilda. Staphylinidae (89 spp.; 53,0\%; excluindo-se Pselaphinae), Carabidae (15 spp.; 8,9\%), Corylophidae (15 spp.; 8,9\%) e Coccinellidae (9 spp.; $5,4 \%$ ) foram os táxons mais representativos para os predadores com $76,2 \%$ do total de morfoespécies (Tabela 1). Tenebrionidae (33 spp.; 45,2\%), Ptiliidae (18 spp.; 24,6\%), Nitidulidae (14 spp.; 19,2\%) e Anthicidae (4 spp.; 5,5\%), corresponderam a 94,5\% do total de espécies dentre os saprófagos, enquanto Pselaphinae (13 spp.; 31,7\%), Endomychidae (10 spp.; 24,4\%), Ciidae e

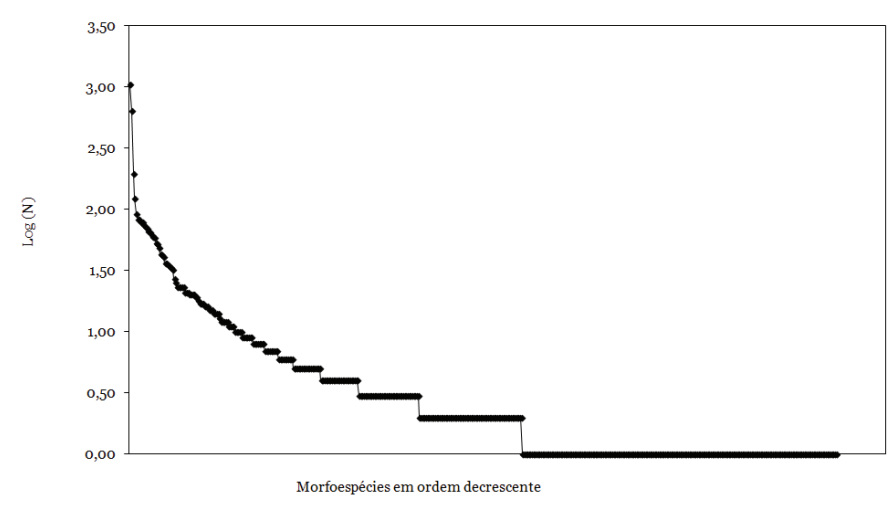

Figura 2. Riqueza de espécies ("Whittaker rank-abundance plot") para a comunidade de Coleoptera (adultos) em copas de A. phalerata Mart. (Arecaceae), durante a fase aquática no Pantanal de Mato Grosso.

Lathridiidae, ambos com cinco morfoespécies (12,2\% cada), compreenderam $80,5 \%$ do total dentre os fungívoros (Tabela 1).

Os 3.132 coleópteros adultos obtidos nas palmeiras I, IV e V, sorteados para o estudo da biomassa, tiveram um peso seco total de 3,8088 g correspondendo a uma biomassa de 0,0762 g/ $\mathrm{m}^{2}$. Scarabaeidae $\left(45,9 \% ; 0,0349 \mathrm{~g} / \mathrm{m}^{2}\right)$, Tenebrionidae $(41,2 \%$; $\left.0,0314 \mathrm{~g} / \mathrm{m}^{2}\right)$, Nitidulidae $\left(1,8 \%\right.$; $\left.0,0014 \mathrm{~g} / \mathrm{m}^{2}\right)$ e Curculionidae $\left(1,7 \%\right.$; $\left.0,0032 \mathrm{~g} / \mathrm{m}^{2}\right)$ compreenderam as famílias com maior biomassa. Das 43 famílias avaliadas, 16 aparecem com valores abaixo de $0,1 \%$. Através da análise das guildas tróficas das famílias de Coleoptera em relação à biomassa $\left(\mathrm{g} / \mathrm{m}^{2}\right)$, verificouse a dominância de herbívoros (51,3\%; 0,0391 g/m²) sobre saprófagos $\left(43,2 \%\right.$; 0,0329 $\left.\mathrm{g} / \mathrm{m}^{2}\right)$, predadores $(4,3 \% ; 0,0033$ $\left.\mathrm{g} / \mathrm{m}^{2}\right)$ e fungívoros $\left(1,1 \% ; 0,0009 \mathrm{~g} / \mathrm{m}^{2}\right)$. A análise de variância evidenciou que a distribuição da biomassa nas três palmeiras avaliadas dentro de cada agrupamento trófico possui diferenças significativas somente para herbívoros $(\mathrm{f}=8400 ; \mathrm{p}=0,001)$ e saprófagos ( $\mathrm{f}=3604 ; \mathrm{p}=0,035)$.

Dentre os herbívoros destacaram-se Scarabaeidae (84,3\%; $\left.0,0349 \mathrm{~g} / \mathrm{m}^{2}\right)$ e Curculionidae $\left(7,5 \% ; 0,0032 \mathrm{~g} / \mathrm{m}^{2}\right)$, enquanto Tenebrionidae (95,3\%; 0,0314 $\left.\mathrm{g} / \mathrm{m}^{2}\right)$ predominou dentre os saprófagos. Os predadores, segundo maior agrupamento em número de espécies, foram representados acentuadamente por Carabidae (31,3\%; 0,0010 g/m²), Lampyridae (29,5\%; 0,0009 g/ $\left.\mathrm{m}^{2}\right)$ e Staphylinidae $\left(21,9 \% ; 0,0007 \mathrm{~g} / \mathrm{m}^{2}\right)$. Dentre os fungívoros, houve predomínio de Endomychidae (65,6\%; 0,0006 g/m²) e Lathridiidae $\left(17,2 \% ; 0,0001 \mathrm{~g} / \mathrm{m}^{2}\right)$.

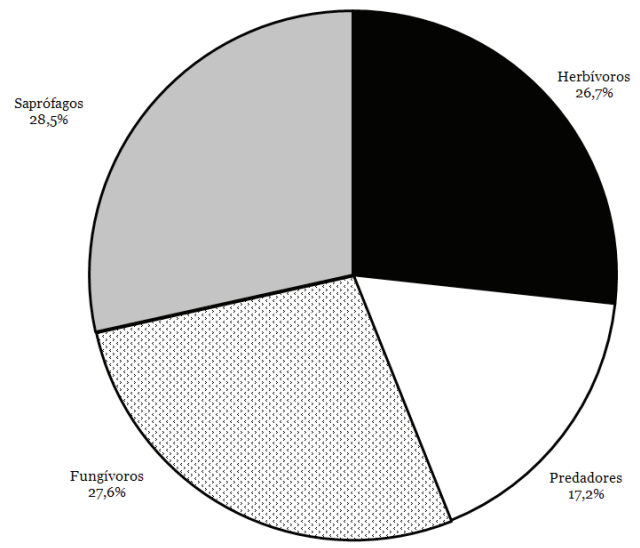

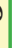

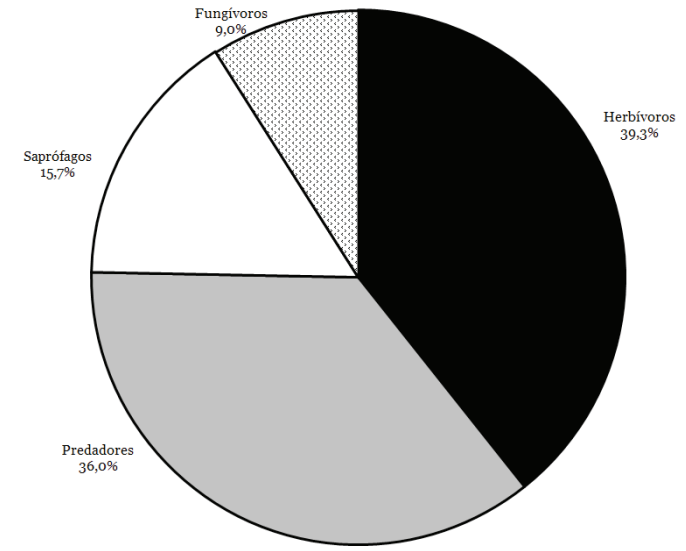

Figura 3. Guildas tróficas das famílias de Coleoptera (adultos) obtidas em copas de A. phalerata Mart. (Arecaceae) no Pantanal de Poconé, Mato Grosso, utilizando como parâmetro de comparação o número de indivíduos (A) e espécies coletadas (B). 
Tabela 2. Número de indivíduos (N), proporção (\%) de Curculionidae (adultos) obtidos em copas de A. phalerata no Pantanal de Poconé, Mato Grosso, segundo a classificação de Alonso-ZARAZAGa \& Lyal (1999), incluindo-se Scolytinae e Platypodinae.

\begin{tabular}{|c|c|c|}
\hline Subfamílias/Tribos/Gênero/Espécie & $\mathbf{N}$ & $\%$ \\
\hline \multicolumn{3}{|l|}{ Baridinae, Baridini, Coelonertina } \\
\hline Coelonertina sp. 18 & 1 & 0,3 \\
\hline Baridini sp.o8 & 2 & 0,6 \\
\hline Baridini sp.11 & 1 & 0,3 \\
\hline Baridini sp.12 & 2 & 0,6 \\
\hline Baridini sp.18 & 4 & 1,1 \\
\hline \multicolumn{3}{|l|}{ Baridinae, Madarini } \\
\hline Notesiaspis hystrix Casey, 1922 & 1 & 0,3 \\
\hline Madarini sp.01 & 1 & 0,3 \\
\hline Madarini sp.06 & 1 & 0,3 \\
\hline Madarini sp.28 & 4 & 1,1 \\
\hline \multicolumn{3}{|l|}{ Ceutorhynchinae } \\
\hline Ceutorhynchinae sp.o1 & 6 & 1,7 \\
\hline Ceutorhynchinae sp.02 & 5 & 1,4 \\
\hline \multicolumn{3}{|l|}{ Conoderinae, Lechriopini } \\
\hline Lechriopini sp.17 & 1 & 0,3 \\
\hline \multicolumn{3}{|l|}{ Cossoninae } \\
\hline Cossoninae sp.o1 & 1 & 0,3 \\
\hline Cossoninae sp.o3 & 3 & 0,8 \\
\hline Cossoninae sp.04 & 1 & 0,3 \\
\hline Cossoninae sp.o5 & 1 & 0,3 \\
\hline \multicolumn{3}{|l|}{ Cryptorhynchinae } \\
\hline Cryptorhynchinae sp.15 & 3 & 0,8 \\
\hline Cryptorhynchinae sp.30 & 1 & 0,3 \\
\hline \multicolumn{3}{|l|}{ Curculioninae, Derelomini } \\
\hline Andranthobius sp.o1 & 4 & 1,1 \\
\hline Andranthobius sp.02 & 58 & 16,2 \\
\hline Andranthobius sp.o4 & 1 & 0,3 \\
\hline Andranthobius sp.o6 & 1 & 0,3 \\
\hline Celetes sp.01 & 1 & 0,3 \\
\hline Derelomini sp.o2 & 1 & 0,3 \\
\hline Derelomini sp.o3 & 6 & 1,7 \\
\hline Derelomini sp.04 & 1 & 0,3 \\
\hline Derelomini sp.10 & 11 & 3,1 \\
\hline Derelomini sp.15 & 2 & 0,6 \\
\hline Derelomini sp.16 & 1 & 0,3 \\
\hline Derelomini sp.18 & 1 & 0,3 \\
\hline Derelomini sp.19 & 2 & 0,6 \\
\hline Derelomini sp.20 & 1 & 0,3 \\
\hline Derelomini sp.21 & 8 & 2,2 \\
\hline Derelomini sp.22 & 3 & 0,8 \\
\hline
\end{tabular}

DISCUSSÃO

Estudos abordando as interações entre palmeiras e invertebrados objetivam avaliar, principalmente, espécies-praga (GIBLIN-DAVIS 2001; Howard 2001; MoOre 2001; Delgado 2002; Cysne et al. 2013), atividade de polinizadores (KAHN \& DE GRANVILLE 1995; Küchmeister et al. 1998; Listabarth 1999; Dulmen 2001; QuirogaCASTRO \& RoldÁn 2001), espécies ou comunidades específicas (Costa et al. 1992; VAsConcelos 1990; SAntos et al. 2003; Battirola et al. 2004a, 2004b, 2005, 2007; GURGEL-GONÇALVEs et al. 2006).

SANTos et al. (2003) analisaram a composição da comunidade de Coleoptera associada à copa de $A$. phalerata nesta mesma região durante o período seca e obtiveram 4.715 coleópteros adultos $\left(65,5\right.$ ind. $\left./ \mathrm{m}^{2}\right)$ distribuídos em 43 famílias e 326 morfoespécies. As famílias mais abundantes, Tenebrionidae, Curculionidae, incluindo Scolytinae e Platypodinae, Carabidae e Staphylinidae representaram juntas, 66,7\% do total coletado. Curculionidae, Staphylinidae e Chrysomelidae corresponderam às de maior número de morfoespécies durante este período sazonal. Esta comparação permite avaliar a variação temporal da composição da comunidade de Coleoptera associada à copa desta palmeira. Para a riqueza da comunidade observa-se um aumento de 43,2\%

\begin{tabular}{|c|c|c|}
\hline Subfamílias/Tribos/Gênero/Espécie & $\mathbf{N}$ & $\%$ \\
\hline \multicolumn{3}{|l|}{ Curculioninae, Tychiini } \\
\hline Tychiini sp.o6 & 1 & 0,3 \\
\hline Tychiini sp.10 & 2 & 0,6 \\
\hline Tychiini sp.11 & 1 & 0,3 \\
\hline \multicolumn{3}{|l|}{ Dryophthorinae $^{1}$} \\
\hline Rhynchophorini sp.o1 & 1 & 0,3 \\
\hline \multicolumn{3}{|l|}{ Entiminae, Naupactini } \\
\hline Naupactini sp.o3 & 2 & 0,6 \\
\hline Naupactini sp.o8 & 1 & 0,3 \\
\hline Naupactini sp.o9 & 1 & 0,3 \\
\hline \multicolumn{3}{|l|}{ Erirhininae $^{2}$, Stenopelmini } \\
\hline Cyrtobagous singularis Hustache & 1 & 0,3 \\
\hline Stenopelmini sp.02 & 1 & 0,3 \\
\hline Stenopelmini sp.12 & 1 & 0,3 \\
\hline Stenopelmini sp.14 & 1 & 0,3 \\
\hline Stenopelmini sp.33 & 6 & 1,7 \\
\hline Stenopelmini sp.42 & 1 & 0,3 \\
\hline \multicolumn{3}{|l|}{ Molytinae } \\
\hline Molytinae sp.05 & 1 & 0,3 \\
\hline Molytinae sp.22 & 1 & 0,3 \\
\hline Molytinae sp.31 & 2 & 0,6 \\
\hline \multicolumn{3}{|l|}{ Molytinae, Conotrachelini } \\
\hline Conotrachelus sp.26 & 4 & 1,1 \\
\hline \multicolumn{3}{|l|}{ Platypodinae } \\
\hline Platypodinae sp.01 & 1 & 0,3 \\
\hline Platypodinae sp.04 & 2 & 0,6 \\
\hline Platypodinae sp.o8 & 1 & 0,3 \\
\hline \multicolumn{3}{|l|}{ Rhythirrininae $^{3}$, Listroderini } \\
\hline Listroderina sp.o8 & 1 & 0,3 \\
\hline Scolytinae (22 morfoespécies) & 178 & 49,7 \\
\hline \multicolumn{3}{|l|}{ Outros } \\
\hline Curculionidae sp.o4 & 2 & 0,6 \\
\hline Curculionidae sp.07 & 1 & 0,3 \\
\hline Curculionidae sp.o8 & 1 & 0,3 \\
\hline Curculionidae sp.09 & 2 & 0,6 \\
\hline Total & 358 & 100,0 \\
\hline
\end{tabular}

1, 2, 3 Subfamílias conforme proposto por MARVALDI \& LANTERI (2005).

no número de espécies do período de seca (326 spp.) para a cheia (467 spp.). Quando se comparam as morfoespécies constata-se que apenas 112 são comuns aos dois períodos, 214 restritas à seca, e 355 à cheia.

Ainda nesta mesma regiãoestudos com nebulização decopas foram desenvolvidos com Vochysia divergens Pohl. (Vochysiaceae) (Marques et al. 2001, 2006) e Calophyllum brasiliense Cambess. (Guttiferae) (MARQuEs et al. 2007) evidenciando que a composição das comunidades arborícolas é variável entre os hospedeiros vegetais em uma mesma região, pois, dentre os táxons obtidos nestes estudos, destaca-se o predomínio de Meloidae e Dryopidae sobre $V$.divergens e de Nitidulidae, Curculionidae e Staphylinidae em copas de C. brasiliense.

Estudos realizados com comunidades arborícolas em diferentes regiões tropicais e diferentes hospedeiros vegetais mostram que as famílias dominantes neste habitat demonstram similaridades, pois, grupos como Curculionidae, Chrysomelidae, Staphylinidae e Tenebrionidae são recorrentes na dominância destas comunidades. DAvies et al. (1997) amostraram seis espécies vegetais na Venezuela obtendo 65 famílias e 978 morfoespécies de Coleoptera. Destas, Curculionidae, Staphylinidae, Anobiidae e Coccinellidae foram dominantes em número de espécies, 
enquanto Chrysomelidae: Bruchinae (14,7\%) predominou com relação ao número de indivíduos. Ainda na Venezuela, KIRMSE et al. (2003) observaram a maior abundância e riqueza distribuída entre Curculionidae, Cerambycidae, Chrysomelidae e Elateridae. No Panamá, ØDEGAARD (2003) obteve Curculionidae e Chrysomelidae como predominantes, tanto em riqueza de espécies, quanto em abundância de indivíduos.

Nos estudos de WAGner (2000) realizados naÁfrica, Staphylinidae, Curculionidae e Chrysomelidae corresponderam aos táxons com maior riqueza de espécies, e as famílias mais abundantes foram Lathridiidae, Chrysomelidae, excluindo-se Bruchinae, e Staphylinidae. Staphylinidae, Chrysomelidae e Curculionidae também representaram as famílias mais ricas em amostras efetuadas em Nova Guiné e Papua Nova Guiné (Allison et al. 1993, 1997). Resultados similares foram obtidos na Amazônia (Erwin 1983), em Borneo (STORK 1991) e na Malásia (Floren \& LinSENMAIR 1998a). Curculionidae e Staphylinidae destacaramse também devido a riqueza de espécies em florestas úmidas de Sulawesi, juntamente com Anthribidae, Aderidae e Corylophidae (HAMmond et al. 1997).

Os curculionídeos (exceto Platypodinae) representam um importante grupo de besouros herbívoros, geralmente citados como dominantes em abundância e diversidade em copas de árvores de florestas tropicais. Para os Derelomini, grupo representativo em copas de $A$. phalerata (Tabela 2), presume-se a função depolinizadores para os adultos, pois, segundo KücHMEISTER et al. (1998) estão, geralmente, associados às inflorescências das palmeiras, local utilizado para o seu desenvolvimento larval. Porém, em algumas espécies o empupamento, aparentemente, ocorre no solo, em lugares sombreados debaixo da planta hospedeira.

Vários Cossoninae, Cryptorrhynchinae e Dryophthorinae estão associados com monocotiledôneas, os primeiros utilizando tecidos vegetais mortos, e o último com suas larvas alimentandose em seus caules e raízes (LAWrence et al. 2000). Tychiini (Curculioninae) também utiliza as estruturas florais para o desenvolvimento larval, porém, a fase pupal pode ocorrer no solo, caso as flores e os frutos caiam antes de sua emergência. Naupactini (Entiminae) passa a fase de larva e pupa no solo, e quando adulto sobre os hospedeiros vegetais, ao contrário de Baridinae, que em sua maioria, possui todo o seu desenvolvimento sobre as plantas, sem contato algum com o solo. Grupos associados às flores e sementes como Baridinae e Molytinae (Anthonomini), possuem um grande número de espécies. Porém, os Baridinae são dificilmente coletados através de nebulização, pois, podem ficar presos aos troncos, galhos e folhas devido aos espinhos existentes em suas tíbias e cavidades ventrais, contribuindo para que a riqueza desta subfamília seja subestimada em amostragens em áreas tropicais (ØDEGAARD 2003).

Os Erirhininae (Stenopelmini) possuem espécies aquáticas e subaquáticas. Nas áreas inundáveis do Pantanal devido à grande abundância de macrófitas aquáticas, é provavel que estes curculionídeos desloquem-se para outros hospedeiros vegetais (LAWRENCE et al. 2000). Outro grupo que pode ser associado troficamente às plantas aquáticas são os Ceutorrhynchinae, tendo sido amostrados como visitantes ocasionais em palmeiras (Tabela 2), influenciando a composição da comunidade neste habitat.

Segundo FARREL \& ERWIN (1988), a arquitetura da copa é um dado a ser considerado em relação à comunidade associada, pois influencia fortemente a riqueza e abundância de alguns grupos como Staphylinidae. Estes autores salientaram ainda que a classificação da arquitetura da copa é um fator determinante à diversidade de Staphylinidae e de outros grupos de predadores, exercendo mais influência que a composição florística de determinada área.
Outra variável citada como determinante para os padrões de diversidade de artrópodes, dentre estes os Coleoptera, é a relação existente entre a fauna de solo e copa (PAARMAnN \& STORK 1987; ADIs 1997), bem como a associação desses organismos com a matéria orgânica acumulada sobre as copas (LoNGINO \& NADKARNI 1990; SANTOS et al. 2003; BatTirola et al. 2004a, 2004b, 2005, 2007; MARQuEs et al. 2009). Estudos realizados na Amazônia por ADIs (1997) indicaram que alguns grupos como Curculionidae e Chrysomelidae, em sua fase larval, alimentam-se no solo sobre raízes e migram, posteriormente, para as copas na fase adulta, ou migram do solo para copa em áreas sujeitas a inundações.

A disponibilidade de recursos tanto no solo, quanto na copa, também exerce papel determinante para a estruturação das comunidades de insetos. Exemplo disto é a dominância de Endomychidae obtida nesta pesquisa. Os Endomychidae são fungívoros que encontram recursos alimentares em abundância nas copas de $A$. phalerata, pois, a alta temperatura associada à umidade constante favorece a proliferação dos fungos neste habitat. Tal fato é possível devido à disponibilidade de matéria orgânica acumulada nas bainhas remanescentes das folhas de A. phalerata, que propiciam um habitat favorável, oferecendo alimento e abrigo, conforme discutido por MARQues et al. (2009). Esta mesma matéria orgânica pode ser responsável pela elevada densidade de larvas de Coleoptera $(34,3 \%$, da captura total de coleópteros) obtida neste estudo.

Com relação à distribuição da comunidade em guildas tróficas em que se observou a predominância de saprófagos, fungívoros e herbívoros sobre predadores observam-se resultados diferenciados dos obtidos por SANTOs et al. (2003) durante o período de seca sobre esta mesma palmeira em que herbívoros e predadores predominaram sobre fungívoros e saprófagos. Essa diferença na estrutura da comunidade pode estar associada à disponibilidade de recursos em cada uma das fases hidrológicas típicas dessa região, embora os grupos dominantes em cada guilda trófica sejam basicamente os mesmos.

Marques et al. (2001), analisando a copa de um indivíduo de $V$. divergens durante a fase terrestre, também no Pantanal matogrossense, obtiveram dominância de herbívoros sobre xilófagos, saprófagos, predadores e fungívoros. Em estudo posterior, em três copas desta mesma espécie arbórea nas fases terrestre e aquática, MARQUES et al. (2006) encontraram predominância de herbívoros e predadores, sobre saprófagos e fungívoros. Estudos conduzidos em outras áreas e formações vegetacionais como na Venezuela obtiveram também a predominância de herbívoros, porém, seguidos por fungívoros (DAviEs et al. 1997). O mesmo ocorreu em Ruanda, em que os herbívoros e fungívoros predominaram sobre outros grupos (WAGNER 1997).

Diferentemente de todos os resultados acima citados, HAmmond (1990) e HAMmond et al. (1996) verificaram em suas amostragens em copas de árvores na Austrália e Sulawesi, respectivamente, a dominância de predadores, principalmente, Staphylinidae e Melyridae, sobre outros grupos tróficos. O predomínio de algumas famílias dentro de seu agrupamento trófico como Tenebrionidae dentre os saprófagos foi constatado por ERwin (1983) em diferentes tipos de florestas na Amazônia. O mesmo ocorreu para Endomychidae e Anthribidae entre os fungívoros, Coccinellidae e Staphylinidae entre os predadores, além de Curculionidae e Chrysomelidae entre os herbívoros, demonstrando similaridade com os padrões observados neste estudo em copas de $A$. phalerata.

A riqueza de herbívoros obtida em copas $A$. phalerata pode estar associada à teoria de concentração de recursos (SouTHwood 1961; Rоот 1973), em que se prevê que plantas com alta densidade são facilmente encontradas por insetos herbívoros, que, consequentemente, permanecem por um maior período de tempo sobre as mesmas, como pode ocorrer em formações monodominantes como os acurizais (adensamentos de A. 
phalerata) no Pantanal de Mato Grosso. Esta situação pode favorecer o aumento dessas populações, e, por sua vez, reduzir as de outros grupos, ocasionando a diminuição da riqueza de espécies na comunidade (MARQues et al. 2000).

Quando comparados os resultados da biomassa e riqueza de espécies verifica-se que os predadores possuem uma baixa biomassa (0,0033 g/m²) associada a uma elevada riqueza (126 espécies), enquanto os saprófagos, uma considerável biomassa $\left(0,0329 \mathrm{~g} / \mathrm{m}^{2}\right)$ com um baixo número de espécies (6o spp.). Isso demonstra que a distribuição da biomassa é bastante heterogênea na comunidade sendo a maior parte restrita a poucos grupos dentro de cada agrupamento trófico. No caso dos herbívoros e fungívoros, os valores de biomassa acompanham a riqueza de espécies, possuindo os primeiros, elevados valores para ambos, e o segundo, reduzidos valores.

A comunidade de Coleoptera associada à copa de $A$. phalerata é rica e diversificada tanto em relação ao número de famílias, quanto à riqueza de espécies. Os agrupamentos em guildas tróficas demonstraram predomínio de saprófagos, fungívoros e herbívoros sobre predadores. A dominância de saprófagos e fungívoros pode estar associada ao acúmulo de matéria orgânica nas bainhas de $A$. phalerata, local propício para desenvolvimento dessas populações, influenciando a constituição e estrutura dessa comunidade. Esses resultados indicam que $A$. phalerata pode ser considerada uma espécie-chave nos ecossistemas inundáveis do Pantanal mato-grossense por desempenhar importante papel nos processos ecológicos destas áreas como local de refúgio e reprodução para diversos grupos de artrópodes, dentre eles os Coleoptera.

\section{AGRADECIMENTOS}

Ao Instituto Max-Planck para Limnologia, Plön, Alemanha e a Universidade Federal de Mato Grosso - Cuiabá, Programa SHIFT (Studies of Human Impact on Forests and Floodplains in the Tropics), financiado pelo Bundesministerium für Bildung, Wissenschaft Forschung und Technologie (BMBF), Conselho Nacional de Desenvolvimento Cientifico e Tecnológico (CNPq) e Instituto Brasileiro de Meio Ambiente e Recursos Naturais Renováveis (IBAMA) e ao Programa de Pós-Graduação em Ecologia e Conservação da Biodiversidade do Instituto de Biociências da UFMT.

\section{REFERÊNCIAS}

Adis, J., 1982. Zur Besiedlung zentralamazonischer Überschwemmungswälder (Várzea-Gebiet) durch Carabiden (Coleoptera). Archives Hydrobiologie, 95: 3-15.

Adis, J., 1988. On the abundance and density of terrestrial arthropods in Central Amazonian dryland forest. Journal of Tropical Ecology, 4: 19-24.

Adis, J., 1997. Terrestrial invertebrates: survival strategies, group spectrum, dominance and activity patterns, p. 299-317. In: Junk, W. J. (Org.). The Central Amazon Floodplain - Ecology of a pulsing system. Ecological Studies 126. Berlin, SpringerVerlag, xxiii +525 .

Adis, J., T.L. Erwin, L.D. Battirola \& S.M. Ketelhut, 2011. The importance of Amazonian floodplain forests for animal biodiversity: Beetles in canopies of floodplain and upland forests, p.313-325In:Junk, W.J., M.T.F.Piedade, F. Wittmann, J. Schöngart \& P. Parolin (Org.). Amazon Floodplain Forests: Ecophysiology, Biodiversity and Sustainable Management. 1ed. Dordrecht: Springer.

Adis, J., Y. Basset, A. Floren, W. Hammond \& K.E. Linsenmair, 1998. Canopy fogging of an overstory tree - recommendations for standardization. Ecotropica 4: 93-97.

Adis, J., Y.D. Lubin \& G.G. Montgomery, 1984. Arthropods from the canopy of inundated and terra firme forests near Manaus, Brazil, with critical considerations on the pyrethrum-fogging technique. Studies on Neotropical Fauna \& Environment, 19:
223-236.

Allison, A., A. Samuelson \& S.E. Miller, 1993. Patterns of beetle species diversity in New Guinea rainforest as revealed by canopy fogging: preliminary findings. Selbyana, 14: 16-20.

Allison, A., A. Samuelson \& S.E. Miller, 1997. Patterns of beetles species diversity in Castanopsis acuminatissima (Fagaceae) trees studied with canopy fogging in mid-montane New Guinea rainforest, p. 224-236. In: Stork. N. E., J. Adis \& Didham, R. K. (Orgs.), Canopy Arthropods. London, Chapman \& Hall, 567.

Alonso-Zarazaga, M.A. \& H.C. Lyal, 1999. A world catalogue of Families and Genera of Curculionoidea (Insecta: Coleoptera) (Excepting Scolytidae and Platypodidae. Entomopraxis, S. C. P., Barcelona. 315p.

Arnett, R.H.Jr., 1963. The beetles of the United States. Washington, D.C.: The Catholic University of America Press, $\mathrm{xi}+1112 \mathrm{p}$.

Barone, J.A., 2000. Comparison of herbivores and herbivory in the canopy and understory for two tropical tree species. Biotropica, 32: 307-317.

Basset, Y. \& G.A. Samuelson, 1996. Ecological characteristics of an arboreal community of Chrysomelidae in Papua New Guinea, p. 243-262. In: Jolivet P.H.A. \& M.I. Cox (Orgs.). Chrysomelidae Biology. v. 2; Ecological Studies. SPB Academic Publishing, Amsterdam.

Basset, Y., 1997. Species abundance and body size relationships in insect herbivores associated with New Guinea forest trees, with particular reference to insect host-specifity, p. 237264. In: Stork, N.E., J. Adis, R.K. Didham (Orgs.). Canopy Arthropods. London, Chapman \& Hall, 567 p.

Basset, Y., G.A. Samuelson, A. Allison \& S.E. Miller. 1996. How many species of hostspecific insects feed on a species of tropical tree? Biological Journal of the Linnean Society 59: 201-216.

Basset, Y., N.D. Springate \& G.A. Samuelson, 1994. Feeding habits and range of body size: a case study in Papua New Guinea using arboreal leaf-beetles and weevils (Coleoptera: Chrysomelidae, Curculionidae). Bulletin de la Societé Entomologique Suisse, 67: 347-361.

Battirola, L.D., G.B. Santos, M.I. Marques \& J. Adis, 2004b. Arthropods from the canopy of Attalea phalerata Mart. (Arecaceae) in the Pantanal of Mato Grosso, Brazil. ICAN Newsletter, 10: 2-3.

Battirola, L.D., J. Adis, M.I. Marques \& F.H.O. Silva. 2007. Comunidade de artrópodes associados à copas de Attalea phalerata Mart. (Arecaceae) durante o período de cheia no Pantanal de Poconé, MT. Neotropical Entomology, 36: 640651.

Battirola, L.D., M.I. Marques, J. Adis \& A.D. Brescovit, 2004a. Aspectos ecológicos da comunidade de Araneae (Arthropoda, Arachnida) em copas de Attalea phalerata Mart. (Arecaceae) no Pantanal de Poconé, Mato Grosso, Brasil. Revista Brasileira de Entomologia, 48: 421-430.

Battirola, L.D., M.I. Marques, J. Adis \& J.H.C. Delabie. 2005. Composição da comunidade de Formicidae (Insecta, Hymenoptera) em copas de Attalea phalerata Mart. (Arecaceae) no Pantanal de Poconé, Mato Grosso, Brasil. Revista Brasileira de Entomologia, 49: 107-117.

Charles, E. \& Y. Basset, 2005. Vertical stratification of leaf-beetle assemblages (Coleoptera: Chrysomelidae) in two forest types in Panama. Journal of Tropical Ecology, 21: 329-336.

Colwell, R.K., 200o. Statistical estimation of species richness and shared species from samples (EstimateS 6.0), 2000.

Costa, E.C., D. Link, G.H. Rosado-Neto, A.D. Grutzmacher \& J.A.S. França, 1992. Entomofauna de inflorescência de palmáceas: 1. Curculionidae (Coleoptera). Anais do $9^{\circ}$ de Congresso de Entomologia 2: 949-954.

Cysne, A.Q., Cruz, B.A., Cunha, R.N.V. \& R.N.C. Rocha, 2013. Flutuação populacional de Rhynchophorus palmarum (L.) (Coleoptera: Curculionidae) em palmeiras oleíferas no Amazonas. Acta Amazônica 43: 197 - 202. 
Davies, J.G., N.E. Stork, M.J.D. Brendell \& S.J. Hine, 1997 Beetle species diversity and faunal similarity in Venezuelan rainforest tree canopies, p. 85-103. In: Stork, N.E., J. Adis \& R.K. Didham (Orgs.). Canopy Arthropods. London, Chapman \& Hall, $567 \mathrm{p}$.

Delgado, C., 2002. The relationship between Pachymerus cardo (Fahraeus) (Coleoptera: Bruchidae) and the palm Orbignya spectabilis (C. Martius) Burret (Arecaceae: Cocoeae) in a the terra firme forest, Brazilian Amazon. Amazoniana 17(1/2): 169-172.

Dulmen, A. V., 2001. Pollination and phenology of flowers in the canopy of two contrasting rain forest types in Amazonia, Colombia, p. 73-85. In: Linsenmair, K.E., A.J. Davies, B. Fiala \& M.R. Speight (eds.). Tropical forest canopies: ecology and management. London, Kluwer Academic Publisher, 370 p.

Endress, P.K., 1994. Diversity and evolutionary biology of tropical flowers. Cambridge Tropical Biology Series, $511 \mathrm{p}$.

Erwin, T.L. 1983. Beetles and other insects of tropical forest canopies at Manaus, Brazil, sampled by insecticidal fogging, p. 59-75. In: Sutton, S.L., T.C. Whitmore \& A.C. Chadwick, (Orgs.). Tropical Rainforest: Ecology and Management. Blackwell Scientific Publications. Oxford, 498 p.

Erwin, T.L. \& J. Adis, 1982. Amazonian inundation forest their role as short-term refuges and generators of species richness and taxon pulses, p. 358-371. In: Prance, G. (Org.). Biological diversification in the tropics. Columbia University Press, New York, $714 \mathrm{p}$.

Erwin, T.L. \& J.C. Scott, 1980. Seasonal and size patterns, trophic structure and richness of Coleoptera in the tropical arboreal ecosystem: the fauna of the tree Luehea seemannii Triana and Planch in the Canal Zone of Panamá. The Coleopterists Bullettin, 34: 305-322.

Farrell, B.D. \& T.L. Erwin, 1988. Leaf-Beetle community structure in an Amazonian rainforest canopy, p. 73-90. In: Jolivet P., E. Petitpieree \& T.H. Hsiao (Orgs.). Biology of Chrysomelidae. Dordrecht, Kluwer Academic Publishers, xxiv + 615 p.

Floren, A. \& K.E. Linsenmair, 1997. Diversity and recolonization dynamics of selected arthropod groups on different tree species in a lowland rainforest in Sabah, Malaysia, with special reference to Formicidae, p. 344-381. In: Stork, N.E., J. Adis \& R.K. Didham (Orgs.). Canopy Arthropods. London, Chapman \& Hall, $567 \mathrm{p}$.

Floren, A. \& K.E. Linsenmair, 1998a. Non-equilibrium communities of Coleoptera in trees in a lowland rain forest of Borneo. Ecotropica, 4: 55-67.

Floren, A. \& K.E. Linsenmair, 1998b. Diversity and recolonization of arboreal Formicidae and Coleoptera in a lowland rain forest in Sabah, Malaysia. Selbyana, 19: 155-161.

Giblin-Davis, R.M., 2001. Borers of palms, p. 267-304. In: Howard, F.W., D. Moore \& R.M. Giblin-Davis (Orgs.). Insects on Palms. Cabi Publishing, New York. 400 p.

Gonçalves, T.T. \& J.N.C. Louzada, 2005. Estratificação vertical de coleópteros carpófilos (Insecta: Coleoptera) em fragmentos florestais do sul do Estado de Minas Gerais, Brasil. Ecología Austral, 15: 101-110.

Gurgel-Gonçalves, R., Palma, A.R.T., Motta, P.C., Bar, M.E. \& C.A.C. Cuba, 2006. Arthropods associated with the crown of Mauritia flexuosa (Arecaceae) palm trees in three different environments from Brazilian Cerrado. Neotropical Entomology 35: 302-312.

Hammond, P.M., 1990. Insect abundance and diversity in the Dumoga-Bone National Park, N. Sulawesi, with special reference to the beetle fauna of lowland rain forest in Toraut region, p. 197-254. In: Knight, W.J. \& J.D. Holloway (Orgs.). Insects and the rain forest of South East Asia (Wallacea). London, Royal Entomological Society of London, 343 p.

Hammond, P.M., N.E. Stork \& M.J.D. Brendell, 1997. Tree-crown beetles in context: a comparison of canopy and other ecotone assemblages in a lowland tropical forest in Sulawesi, p. 184223. In: Stork, N.E., J. Adis \& R.K. Didham (Orgs.). Canopy Arthropods. London, Chapman \& Hall, 567 p.
Hammond, P.M., R.L. Kitching \& N.E. Stork, 1996. The composition and richness of the tree-crown Coleoptera assemblage in an Australian subtropical forest. Ecotropica, 2: 99-108.

Heckman, C.W., 1998. The Pantanal of Poconé. Dordrecht, Kluwer Academic Publishers, 622 p.

Howard, F.W., 2001. The animal class Insecta and the planf family Palmae, p. 1-32. In: Howard, F.W., D. Moore \& R.M. Giblin-Davis (Orgs.). Insects on Palms. Cabi Publishing, New York. 400 p.

Kahn, F. \& J.J. De Granville, 1995. Palms in forest ecosystems of Amazonia. Ecological Studies 95, Berlin, 226 p.

Kirmse, S., J. Adis \& W. Morawetz, 2003. Flowering events and beetle diversity in Venezuela, p. 256-265. In: Basset Y., V. Novotny, S.E. Miller \& R.L. Kitching (Orgs.). Arthropods of Tropical Forests: Spatio-temporal Dynamics and Resource Use in the Canopy. Cambridge, Cambridge University Press, $474 \mathrm{p}$.

Kitching, R.L., H. Mitchell, G. Morse \& G. Thebaud, 1997. Determinants of species richness in assemblages of canopy arthropods in rainforests, p. 131-150. In: Stork, N.E., J. Adis \& R.K. Didham (Orgs.). Canopy Arthropods. London, Chapman \& Hall, 567 p.

Küchmeister, H., A.C. Webber, I. Silberbauer-Gottsberger \& G. Gottsberger, 1998. A polinização e sua relação com a termogênese em espécies de Arecaceae e Annonaceae da Amazônia Central. Acta Amazônica, 28: 217-245.

Lawrence, J.F., A.M. Hastings, M.J. Dallwitz, T.A. Paine \& E.J. Zurcher, 2000. Beetles of the World. A key and information system for families and subfamilies. Version 1.0 MS Windows. Melbourne, Australia. CSIRO Publishing.

Listabarth, C. 1999. Pollination studies of palm populations: a step toward the application of a biological species concept, vol. 83, p. 79-93. In: W.R. Buck \& W.W. Thomas (eds.). Evolution, variation, and classification of palms. New York, The New York Botanic Garden Press, 324 p.

Longino, J.T. \& N.M.A. Nadkarni, 1990. comparison of ground and canopy leaf litter ants (Hymenoptera: Formicidae) in a Neotropical montane forest. Psyche, 81-93.

Marques, E.S.A., P.W. Price, N.S. Cobb, 2000. Resource abundance and insect herbivore diversity on woody fabaceous desert plants. Environmental Entomology, 29: 696-703.

Marques, M.I., G.B. Santos, L.D. Battirola \& A.S.O. Tissiani, 2009. Entomofauna associada à matéria orgânica em bainhas foliares de Attalea phalerata Mart. (Arecaceae) na região norte do Pantanal de Mato Grosso. Acta Biologica Paranaense, 38: 93-112.

Marques, M.I., J. Adis, C. Nunes da Cunha \& G.B. Santos, 2001. Arthropod biodiversity in the canopy of Vochysia divergens Pohl (Vochysiaceae), a forest dominant in the Brazilian Pantanal. Studies on Neotropical Fauna and Environment, 36: 205-210.

Marques, M.I., J. Adis, G.B. Santos \& L.D. Battirola, 2006. Terrestrial arthropods from tree canopies in the Pantanal of Mato Grosso, Brazil. Revista Brasileira de Entomologia, 50: 257-267.

Marques, M.I., J. Adis, L.D. Battirola, A.D. Brescovit, F.H.O. Silva \& J.L. Silva, 2007. Composição da comunidade de artrópodes associada à copa de Calophyllum brasiliense Cambess. (Guttiferae) no Pantanal mato-grossense, Mato Grosso, Brasil. Amazoniana, 19: 131-148.

Marvaldi, A.E. \& A.A. Lanteri, 2005. Key to higher taxa of South American weevils based on adult characters (Coleoptera, Curculionoidea). Revista Chilena de História Natural, 78: 6587.

Mawdsley, N.A. \& N.E. Stork, 1997. Host-specifity and the effective specializacion of tropical canopy beetles, p. 104130. In: Stork, N.E., J. Adis \& R.K. Didham (Orgs.). Canopy Arthropods. London, Chapman \& Hall, 567 p.

Moore, D., 2001. Insects on palm flowers and fruits, p. 233-266. In: Howard, F.W., D. Moore \& R.M. Giblin-Davis (Orgs.). 
Insects on Palms. Cabi Publishing, New York. 400 p.

Novotny, V., Y. Basset, A.G. Samuelson \& S.E. Miller, 1999. Host use by chrysomelid beetles feeding on Moraceae and Euphorbiaceae in New Guinea. p: 343-36o In: Cox, M.L. (Org.). Advances of Chrysomelidae Biology. Backhuys, Leiden.

Novotny, V., Y. Basset, S.E. Miller, G.D. Weiblen, B. Bremer, L. Cizek \& P. Drozd. 2002a. Low host specificity of herbivorous insects in a tropical forest. Nature, 416: 841-844.

Novotny, V., Y. Basset, S.E. Miller, P. Drozd \& L. Cizek, $2002 b$. Host specialization of leaf chewing insects in a New Guinea rainforest. Journal of Animal Ecology, 71: 400-412.

Ødegaard, F., 2003. Taxonomic composition and host specificity of phytophagous beetles in a dry forest in Panama, p. 220236. In: Basset, Y., V. Novotny, S.E. Miller \& R.L. Kitching (Orgs.). Arthropods of Tropical Forests: Spatio-temporal Dynamics and Resource Use in the Canopy. Cambridge, Cambridge University Press, 474 p.

Paarmann, W. \& N.E. Stork, 1987. Canopy fogging, a method of collecting living insects for investigations of life history strategies. Journal of Natural History, 21: 563-566.

Pokon, R., V. Novotny, V. \& G.A. Samuelson, 2005. Host specialization and species richness of root-feeding chrysomelid larvae (Chrysomelidae, Coleoptera) in a New Guinea rain forest. Journal of Tropical Ecology, 21: 595-604.

Quiroga-Castro, V.D. \& A.L. Roldán, 2001. The fate of Attalea phalerata (Palmae) seeds dispersed to a tapir latrine. Biotropica, 33: 472-477.

Root, R.B. 1973. Organization of a plant-association in simple and diverse habitats: the fauna of collards (Brassica oleraceae). Ecological Monographs, 43: 95-124.

Santos, G.B., M.I. Marques, J. Adis \& C.R. De Musis, 2003. Artrópodos associados à copa de Attalea phalerata Mart. (Arecaceae), na região do Pantanal de Poconé-MT. Revista Brasileira de Entomologia, 47: 211-224.
Southwood, T.R.E., 1961. The number of species of insect associated with various trees. Journal of Animal Ecology, 30: 1-8.

SPSS 12.0 Statistical Package for the Social Sciences (SPSS). Version 12.o. Chicago: SPSS Inc.; 2006.

Stork, N.E., 1987. Guild structure of arthropods from Bornean rain forest trees. Ecological Entomology, 12: 69-80.

Stork, N.E., 1991. The composition of arthropod fauna of Bornean lowland rainforest trees. Journal of Tropical Ecology, 7: 161180.

Stork, N.E. \& T.M. Blackburn, 1993. Abundance, body size and biomass of arthropods in tropical forest. Oikos, 67: 483-489.

Vasconcelos, H.L. 1990. Effects of litter collection by understory palms on the associated macroinvertebrate fauna in Central Amazonia. Pedobiologia 34: 157-160.

Wagner, T., 1997. The beetle fauna of different tree species in forests of Rwanda and East Zaire, p. 169-183. In: Stork, N.E., J. Adis \& R.K. Didham (Orgs.). Canopy Arthropods. London, Chapman \& Hall, 567 p.

Wagner, T., 2000. Influence of forest type and tree species on canopy-dwelling beetles in Budongo forest, Uganda. Biotropica, 32: 502-514.

Wagner, T., 2003. Seasonality of canopy beetles in Uganda, p. 146-169. In: Basset, Y., V. Novotny, S.E. Miller \& R.L. Kitching (Orgs.). Arthropods of Tropical Forests: Spatio-temporal Dynamics and Resource Use in the Canopy. Cambridge, Cambridge University Press, 474 p.

\section{Recebido em: 29/01/2013}

Aceito em: 02/12/2013

\section{Como citar este artigo:}

Battirola, L.D., G.B. dos Santos, G.H. Rosado-Neto \& M.I. Marques, 2014. Coleoptera (Arthropoda, Insecta) Associados às Copas de Attalea phalerata Mart. (Arecaceae) no Pantanal de Mato Grosso, Brasil. EntomoBrasilis, 7 (1): 20-28.

Acessível em: doi:10.12741/ebrasilis.v7i1.316
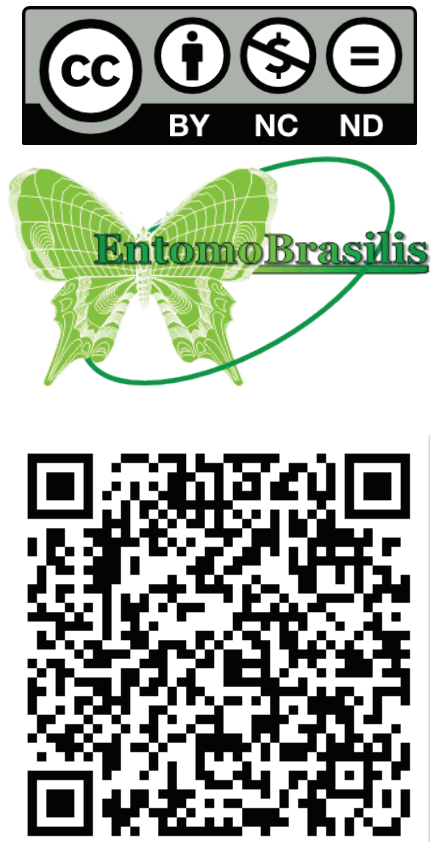